\title{
Digital ethnicity: discussion of a concept and implications for education
}

\author{
Nan B. Adams*, Thomas A. DeVaney \\ Southeastern Louisiana University, Hammond, LA 70204, USA
}

\begin{abstract}
Interaction with the rapidly expanding digital technologies for education, work, and play has drastically changed the processes and practices of world populations. As societies evolve in response to these new communication and calculation tools, the need arises to understand the sometimes unique but increasingly common change in cultures. The Digital Ethnicity Scale (DES), utilizing Longstreet's (1978, Aspects of Ethnicity (New York: Teachers College Press) model of the Aspects of Ethnicity, was developed to describe the emergence of new cultural patterns of behavior that result from the influence of human interaction with digital communication technologies (Adams, DeVaney, and Longstreet (2010) Comput. Hum. Behav. 26(6): 1822-1830). Longstreet's definition of ethnicity focuses on cultural development during the earliest stages of human development, prior to the onset of children's abstract thinking. The ultimate goal for the development of the DES is to describe those aspects of digital ethnicity and collect these descriptions along with demographic data to achieve profiles of various digital ethnicities. A discussion of the guiding concept and overview of the development of the DES seeks to present a description of these digital ethnic profiles that may provide insight into the educational needs of rapidly changing societal groupings with hopes of providing guidance for educational practice.
\end{abstract}

Keywords: computers and human behavior, educational computing, socio-cultural patterns

Received on 6 January 2011; accepted on 12 January 2011

Copyright (C) 2011 Adams and DeVaney, licensed to ICST. This is an open access article distributed under the terms of the Creative Commons Attribution licence (http://creativecommons.org/licences/by/3.0/), which permits unlimited use, distribution and reproduction in any medium so long as the original work is properly cited.

doi: 10.4108/icst.trans.eeel.2011.e2

\section{Introduction}

Digital communication technologies increasingly pervade every facet of our lives. The influence of these technologies is changing human actions and beliefs. Many people would rather 'text' rather than 'talk'; couples often fall in love online and meet after the fact; gender identity is becoming a choice made not by biology but by a screen name or the physical appearance of an avatar. The influence of these changes on the construction of our social reality appears to be in flux. Social groups are formed in virtual space that provides new kinds of common identity to previously disparate individuals. As educators strive to develop meaningful and effective learning strategies, the basic assumptions of how a society thinks and acts along with useful descriptions of those participant in this society

${ }^{*}$ Corresponding author. Email: Nan.Adams@selu.edu are critical to provide context to process and guide educational practice to effect desired outcomes.

Prensky (2001) shares this concern and writes of Digital Natives and Digital Immigrants and how children are being socialized in vastly different ways than their parents. He contends that 'it is now clear that as a result of this ubiquitous [digital] environment and the sheer volume of their interaction with it, today's students think and process information fundamentally differently than their predecessors (2001, para 4)'. He cites the skill of reflection as one key area of loss experienced by children immersed in interaction with digital technologies. He defines reflection as the ability to generalize and create mental models from our experiences and suggests that the implication for this loss is the need to develop educational strategies for developing reflection and critical thinking to counteract this seemingly media-induced loss in children. His most recent writings center on the development of what he terms 
Digital Wisdom (2009), and calls for concerted efforts to realize the impact on child development both intellectually and socially. He argues the need for teaching 'digital wisdom'. He contends that 'the digitally wise distinguish between digital wisdom and mere digital cleverness'. He concludes that 'It is through interaction of the human mind and digital technology that the digitally wise person is coming to be. I believe it is the time for the emerging digitally wise among us ... to embrace digital enhancement and to encourage others to do so (p. 7)'.

Palfrey and Gasser (2008) continue the discussion of the development of 'digital natives' whose 'culture is global in scope and nature. Whether physically based in Rio de Janeiro, Shanghai, Boston, Oslo, or Cape Town, Digital Natives ... form part of global culture of their peers. They are connected to each other in terms of how they relate to information, how they relate to new technologies, and how they relate to one another (p. 13)'. They contend that digital natives are 'quite sophisticated in the ways that they gather information' and portend that 'the people to be worried about are those who are growing up in a digital age but who are not learning these sophisticated information-gathering and informationprocessing skills (p. 241)'.

Small and Vorgan (2008) argue the effects of extensive interaction with digital technologies and contend that 'as the brain evolves and shifts its focus toward new technological skills it drifts away from fundamental social skills, such as reading facial expressions during conversation or grasping the emotional context of a subtle gesture (p. 2)'. They discuss an increasing 'brain gap' (p. 3 ) resulting in separate cultures. They go on to describe changes in communication preference style and the concern that 'some individuals cannot effectively handle the multitasking demands of modern technology, and sometimes syndromes such as attention deficit disorder or attention deficit hyperactivity disorder can result (p. 62)'.

The lives of young children are increasingly influenced by digital technologies, guiding their understanding of the world that surrounds them and influencing their development of communication and language skills, it is vital that deliberate analysis of these developmental changes take place. Engagement in these digital environments starting at an extremely early age is shaping the preferences young children develop for their construction of reality and guides their interaction with their surroundings. The implications for the ever-increasing use of digital communication technologies on current and future society are not clear and the importance of pursuing this topic to guide educational practice is apparent.

The unique construction of the concept of ethnicity originally developed by Longstreet (1978) to describe patterns, or aspects, that represent areas of social behavior that may exist among members of a social group, appears to provide an appropriate and useful framework for investigating the impact digital communication tools are having on cultures and societies. Her definition of ethnicity focuses on cultural development during the earliest stages of human development, prior to the onset of children's abstract thinking. The vast majority of children in today's schools have spent their earliest years heavily influenced by this pervasive digital presence. Additionally, her work was originally based on observations of students in schools. This fact underscores the appropriateness of the Aspects of Ethnicity model to guide inquiry into the educational concerns about this topic.

Longstreet's model (1978) was tested in terms of the digital environment to collect data about the different aspects of ethnicity using a digital lens. The ultimate goal for the development of the Digital Ethnicity Scale (DES) is to describe those aspects of digital ethnicity and collect these descriptions along with demographic data to achieve profiles of various digital ethnicities. These digital ethnic profiles may provide insight into the social and educational needs of rapidly changing societal groupings with hopes of providing guidance for future practicerewrite this after the methodology changes.

\section{Ethnicity-no definitive definition}

The word 'ethnicity' conjures both abstract and concrete meanings, which are often contextual. Governments and institutions often utilize the more concrete meaning, where ethnicity usually denotes race. In the abstract, ethnicity often describes a group of human beings who are identified through shared characteristics that may be real or assumed. This ambiguity reflects the lack of agreement among scholars that has ebbed and flowed along with interest in the endeavor of building a consensus for meaning. Isajiw (1974), a noted anthropologist, analyzed 65 sociological and anthropological studies and found that only 13 had definitions for the term ethnicity, with the remaining 52 having no explicit definition at all. With no real resolution in sight, the term has been defined as needed by institutions and individuals to gather data or describe groups of people.

Two major belief systems inform the issue: objectivists, who define ethnic groups as cultural and social entities with distinct boundaries that are characterized by lack of interaction and relative isolation; while subjectivists describe ethnic groups as culturally-constructed categorizations that guide social behavior and interaction with definition of these groups by subjective self-categorizations (Jones, 1997). This raises the question of whether ethnic groups are based on shared, objective cultural practices that exist independently or the more subjective notion that ethnic groups are constructed by the processes of perception and derived social organization of their members. Another tension exists between specificity and generality; definitions that are too broad to be of any use or definitions so narrow that their usefulness for comparison is minimal. A functional theory of ethnicity must be employed to be of use to the study of ethnicity. 
The subjective, culturally-constructed ethnic groupings employ a scholarly assumption of greater self-awareness than is truly possible at very early stages of human development. To a definition and utilize a model for the concept of ethnicity, Longstreet's (1978) socio-biological model was chosen to guide this inquiry. Her model addresses this assumption by including the consideration for biological human development along with social human development. Children are in the midst of learning abstract symbolic systems, both verbal and nonverbal, without conceptual grasp of the abstractions underlying these systems.

\section{Longstreet's concept of ethnicity}

Longstreet, unlike other scholars, provides the only constructed model for describing identified aspects of ethnicity. This model provides the socio-biological definition of ethnicity as being 'that portion of cultural development that occurs before the individual is in complete command of his or her abstract intellectual powers and that is formed primarily through the individual's early contacts with family, neighbors, friends, teachers, and others as well as with his or her immediate environment of the home and neighborhood (1978, p. 19)'. This construction of the concept of ethnicity, originally developed to describe patterns that may exist among members of a social group, provides an appropriate and useful framework for investigating the impact digital communication tools are having on cultures and societies. Our children are interacting with computers very young, even as early as two or three years of age, which puts them into the age when they are powerful learners of languages of all kinds-including the operational languages of computing. Longstreet's aspects of ethnicity are helpful in describing children growing up engaged and often surrounded by digital environments that encompass their early childhood. They are engaged in interactive video and computer games and other forms of digital communication at a time when biological development and ethnic understandings are most influenced, and yet these young children are not yet in command of their full abstract and intellectual powers and there is a lack of conceptual awareness of what is happening to them. The ultimate goal for the development of the DES is to describe those aspects of ethnicity using a digital lens and collect these descriptions along with demographic data to develop profiles of various digital ethnicities. These digital ethnicity profiles may provide guidance for the social and educational needs of a rapidly changing digital world.

\section{Aspects of ethnicity - the underlying theory and working model}

Longstreet developed a functional model for the five aspects that may be used to describe her concept of ethnicity. These aspects are (i) social value patterns, (ii) intellectual mode, (iii) orientation mode, (iv) verbal communication, and (v) nonverbal communication. A brief description of each follows:

Verbal Communication may be described as the structure a person uses when communicating orally. The rules or patterns for this oral communication are learned by children prior to the development of their abstract intellectual abilities. The ability to learn language seems to be a universal capacity of humankind (Longstreet, 1978, p. 42 ).

Nonverbal Communication may be described as a system of facial expression, body movements, and spatial arrangements that communicate meaning to others (Longstreet, 1978, p. 59).

Orientation Mode refers to patterns of behavior used, regardless of the presence of others, as ways of orienting oneself to the differing contexts of one's usual environment. It may be described as the way one communicates with themselves (Longstreet, 1978, p. 74). The orientation mode may be the most complex of the described modes. This mode is influenced by the social environment but ultimately becomes the ways one becomes comfortable in their own environment when no communication takes place.

Social Value Patterns are based on the sets of persistent behaviors that a group expects from its members and upon which it places certain values and upholds with certain beliefs (Longstreet, 1978, p. 89).

Intellectual Modes are described by Longstreet as the most emotionally charged aspect of ethnicity. This mode is not intended to deal with human innate intelligence, but rather reflect the way we externalize our thoughts, how we approach a problem, what gets our full attention, and what details we are most likely to recall. Intellectual modes link intellectual performance to past experiences (Longstreet, 1978, p. 106-107).

When seeking a model to describe human development through social interaction, and especially social interaction in digital communication environments, the notion of identity often emerges. The authors wish to acknowledge that identity focuses on the individual's definition of self (Erikson, 1968), whereas ethnicity describes an individual's place or believed inclusion within a cultural group. This inquiry focuses on the individuals as they relate to a group.

\section{Digital ethnicity: a specialized form of ethnicity}

When constructing a scale to describe those aspects of ethnicity that may be influenced by early and pervasive interaction with digital communication technologies, consideration of which aspects to investigate was a challenging task. Longstreet predicted a variety of contextual ethnicities that may be distinct ethnicities. These distinct and specialized ethnicities were described as being 
grounded in one or more of the identified five aspects of ethnicity. For example, Scholastic ethnicity may be a distinct form of ethnicity grounded in intellectual mode but still related to and having impact upon the other modes identified within this construction of ethnicity. National ethnicity may be a distinct form of social value patterns and communication modes. Gender ethnicity may be a distinct form of orientation mode. In this vein, the current research has sought to describe Digital Ethnicity as a distinct form of the combined Communication Mode, which is a combination of verbal and nonverbal communication modes.

\section{Measuring digital ethnicity}

Marshall McLuhan observed that 'Everybody experiences far more than he understands. Yet it is experience, rather than understanding, that influences behavior (1964, p. 277)'. As digital communication technologies increasingly replace face-to-face communication and interactions, the experiences that construct human perceptions of reality are altered. The DES seeks to describe those aspects of ethnicity that are influenced by immersive experience with digital communication tools.

Adams et al. (2010) developed the DES to measure, within the context of a digital environment, the aspects of ethnicity proposed by Longstreet (1978). According to Adams et al., the DES development was influenced by the contextual application of the Communication mode aspects, specifically, the combining of nonverbal and verbal communication modes and failure to identify Communication as a unique factor when included in analyses containing the remaining three aspects. The final version of the DES has a two-section structure.

The first section includes 12 statements to which individuals respond using a 4-point Likert-type format ranging from Strongly Agree to Strongly Disagree. A fifth option is also provided that allows the individual to mark I Don't Know. The first section of the DES contains three subscales related to (a) Social Value Patterns, (b) Orientation Mode, and (c) Intellectual Mode. Alpha reliability estimates for the subscales ranged from 0.57 to 0.65 .

As noted above, the first section of the DES addressed three of the five aspects of ethnicity proposed by Longstreet (1978). Adams et al. (2010) noted that within a digital environment, the original aspects of written communication and verbal communication were difficult to separate and proposed a single aspect related to communication mode. According to Adams et al., the aspect of communication mode was originally proposed as an additional subscale in the Likert-type section of the instrument. However, the communication mode items consistently failed to identify a unique factor and loaded across the other three aspects. Consequently, a second section of the DES was created and focused solely on the measurement of the communication mode aspect.
This section includes a set of 16 semantic pairs. For each pair, the members were placed on opposite ends of a 7 -point response field. The individual is instructed to identify his or her feeling or preference about using computers to communicate by marking the desired response option.

\section{Digital ethnicity scale-ongoing refinement}

When constructing a scale to describe those aspects of ethnicity that may be influenced by early and pervasive interaction with digital communication technologies, consideration of which aspects to investigate was challenging. We were unable to obtain consistent separate sets of data for the aspects of verbal and nonverbal communication. It appears that the digital media, not television but all other digital communication environments, have impacted the verbal forms of communication in ways that cannot be tested separately from nonverbal communication and in ways that do not exist in other environments. This fusion may well be a major characteristic of digital ethnicity, but not one that can as yet be characterized by the instrument we have developed. However, digital influences on those ethnic aspects of social value patterns, orientation mode, and intellectual mode provided distinct descriptions of digital ethnic behavior that appear to be useful for the development of an instrument focused on construction of digital ethnic profiles.

Just because this research did not find a description of the changes occurring to communication modes does not mean that these changes are not occurring. McLuhan's conception that the Medium is the Message (1967) provides insight into this media-induced change and is probably more relevant now than when it was originally discussed. Even more relevant may be pursuing the impact of the digital environment on the construction of meaning and even of reality.

\section{Directions for exploration and expansion of the theoretical basis of digital ethnicity}

The use of existing models to explain developing phenomena often serves as the first step of the exploration process. The authors are encouraged by the current findings that surround the concept of Digital Ethnicity. They also wish to note that there exists a large amount of interaction between the world around us and the digital environment-we are currently experiencing multiple environments that the individual is negotiating. For example, we write on a computer, we stop and talk, we may well send copies of the writing back and forth digitally and then discuss the work verbally. The investigation of this negotiation of a variety of environments is not studied with this current inquiry. This should be part of further study, but may be informed by the development of digital ethnic profiles. The ability to understand and 
accommodate changing orientation and intellectual modes along with an understanding of changing social value patterns that result from interaction with digital media will inform educators and other social scientists as we work to understand this emerging digital society. It is possible that a different research methodology will be required to do this proposed future research such as interviews and observations.

\section{Implications of digital ethnicity for educational practice}

Schools have spent the past few decades investing heavily in digital technologies. Computers, Internet access, digital cameras, webcams, computer-based instructional programs, instructional websites, along with texting, chat on PDAs are but a few of the myriad digital teaching tools that are employed in today's classrooms. The basic assumption underlying this change in educational practice is that interaction with these digital teaching tools will facilitate learning and increase educational efficacy. The change in practice was not based on prolific research, but instead on a change in cultures that were rapidly adopting digital communication tools. The mad dash to digital learning continues, but some scholars are beginning to research the actual changes in human development that may be resultant from interaction with digital technologies from a very early age.

Research has begun to show that certain assumptions about the use of digital communication technologies were just that-assumptions. For instance, it was assumed that the use of hypertext would greatly enhance the understanding of readers over the more traditional, linear presentation of information found in books. Hypertext may be defined as the linking of related pieces of information electronically to allow a user quick access to this related information. Hypertext is a common feature in electronic writings that allows the reader to select a word from electronic text and receive additional information pertaining to that word, such as a definition or related references within the text (Encyclopedia Brittanica, 2011). Instead, research shows that the use of hypertext significantly increases the reader's cognitive load and lessens comprehension and retention of the information presented (Neiderhauser et al., 2000; Miall \& Dobson, 2001; DeStafano \& LeFevre, 2007). Carr (2010) observes that readers of hypertext often end up clicking distractedly through pages of text rather than reading for content.

Issues of authority of information sources abound when considering using the Internet. Information Literacy has become a topic that many consider vital to our ability to raise children (or sustain an informed society, for that matter). Because the Internet is not currently filtered for content accuracy, published sites are not required to demonstrate the legitimacy of their information or their claims (ACRL, 2011). The assumption that only truthful information is found on the Internet may not be a good assumption to make.

Schools are institutions developed to educate citizens to assume productive roles in the society in which they live. Those skills deemed necessary and those beliefs and assumptions of organization of that particular society or culture drive educational practice. As schools worldwide rapidly adopt digital technologies for instruction, the result is a more digitally focused society. If the digital fallacies demonstrated by such assumptions as the benefits of hypertext to reading comprehension or that anything found on the web must be true teach us anything, it is that investigation and description is vital to determine the actual changes in society that result from this change in educational practice.

The ultimate goal for the championing of the concept of Digital Ethnicity coupled with the development of the DES is to describe those aspects of digital ethnicity and collect these descriptions along with demographic data to achieve profiles of various digital ethnicities. By describing these digital ethnic profiles, it is expected that some insight into identifying specific educational needs along with guidance for appropriate educational practices of rapidly changing societies may emerge.

\section{References}

ACRL-Association of College and Research Librarians, http://www.ala.org/ala/mgrps/divs/acrl/issues/infolit/ overview/intro/index.cfm (accessed 7 January 2011).

Adams, N.B., DeVaney T.A. and Longstreet, W.S. (2010) Investigating aspects of an emerging digital ethnicity: development of the digital ethnicity scale (DES). Comput. Hum. Behav. 26(6): 1822-1830.

CARr, N. (2010) The Shallows: What the Internet is Doing to Our Brains (New York: W.W. Norton).

DeStefano, D., and LeFevre, J. (2007) Cognitive load in hypertext reading: a review. Comput. Behav. 23(3): 16161641.

Encyclopedia Britannica (accessed 31 May 2011), http:// www.britannica.com/EBchecked/topic/279726/hypertext

Erikson, E. (1968) Identity: Youth and Crisis (New York: W.W. Norton).

IsAJIw, W. (1974) Definitions of ethnicity. Ethnicity 1:111-124.

Jones, S. (1997) The Archeology of Ethnicity (London: Routledge).

Longstreet, W.S. (1978) Aspects of Ethnicity (New York: Teachers College Press).

McLuhan, M. (1964) Understanding Media: The Extensions of Man (New York: McGraw-Hill).

McLuhan, M. (1967) The Medium is the Massage (New York: Bantam Book). 
Miall, D.S. and Dobson, T. (2001) Reading hypertext and the experience of literature. J. Digital Inf. 2(1) http://journals.tdl.org/jodi/article/viewArticle/35/37 (accessed 27 July 2011).

Niederhauser, D.S., Reynolds, R.E., SAlmen, D.J. and SKolmoski, P. (2000) The influence of cognitive load on learning from hypertext. J. Educ. Comput. Res. 23(3): 237-255.

Palfrey, J. and Gasser, U. (2008) Born Digital: Understanding the First Generation of Digital Natives (New York: Basic Books).

Prensky, M. (2001) Digital natives, digital immigrants. On the Horizon 9(5): 1-6. http://www.marcprensky.com/writing/
Prensky\%20-\%20Digital\%20Natives, \%20Digital\%20Immigrants\% 20-\%20Part1.pdf (accessed 8 July 2010).

Prensky, M.H. (2009) Sapiens digital: from digital immigrants and digital natives to digital wisdom. Innovate J. Online Educ. 5(3): 1-9. http://www.innovateonline.info/pdf/ vol5_issue 3/H._Sapiens_Digital__From_Digital_Immigrants_ and_Digital_Natives_to_Digital_Wisdom.pdf (accessed 8 July 2010).

Small, G. and Vorgan, G. (2008) iBrain: Surviving the Technological Alteration of the Modern Mind (New York: Harper Collins Publishers). 